\title{
Postmarketing Safety Profile of Subcutaneous Interferon Beta-1a Given 3 Times Weekly: A Retrospective Administrative Claims Analysis
}

Meredith Y. Smith, PhD, MPA; Meritxell Sabidó-Espin, MD; Anton Trochanov, MD; Mark Samuelson, MD; Sandra Guedes, PharmD; Frank A. Corvino, PhD; and Florent F. Richy, MPH, PhD

\section{ABSTRACT}

BACKGROUND: Health insurance administrative claims databases represent a valuable source of information regarding the safety profile of marketed products as used in actual clinical practice in a broader range of patients than that assessed in clinical trials. Interferon beta-1a administered subcutaneously 3 times weekly (IFN $\beta$-1a SC tiw), which was approved in 2002 by the FDA for the treatment of relapsing-remitting multiple sclerosis (MS), has over a decade of postmarketing experience. To date, however, its postmarketing safety profile has not been described using a real-world evidence source such as administrative claims data.

OBJECTIVE: To describe the safety profile of IFN $\beta-1$ a SC tiw as presented in its U.S. prescribing information (PI) for patients with MS initiating IFN $\beta-1$ a SC tiw therapy using data from U.S. health care administrative claims databases.

METHODS: This study featured an observational and retrospective "new start" cohort design using data from the Truven MarketScan Commercial and Medicare Supplemental health care administrative claims databases. Patients were eligible for inclusion if they were aged $\geq 18$ years; had $\geq 1$ diagnosis for MS recorded between January 1, 2006, and December 31, 2012; had $\geq 2$ prescriptions for IFN $\beta$-1a SC tiw; and had $\geq 90$ days of continuous eligibility pre-index date and $\geq 180$ days of continuous eligibility post-index date. Patients with a prescription for IFN $\beta$-1a SC tiw without a MS diagnosis were excluded. Patients were followed from first prescription for IFN $\beta$-1a SC tiw (index date) until date of therapy switch or discontinuation, end of insurance eligibility, or end of observation period. Adverse events (AEs) examined were those listed in the Warnings and Precautions, Adverse Reactions, and Postmarketing Experience sections of the 2014 U.S. PI. Outcomes of interest were identified at the Medical Dictionary for Regulatory Activities (version 17.1) Preferred Term level and then coded to the corresponding ICD-9-CM criteria. Descriptive analyses of patient demographic, health status, health care utilization, and adherence status were performed, and incidence rates (IRs) per 100 person-years of labeled AEs with corresponding $95 \%$ Cls were calculated. The IR calculation was based on events that presented after therapy initiation and that were not present in the 90-day pre-index period.

RESULTS: The top 6 AEs included influenza-like symptoms (IR $=15.65$, $95 \% \mathrm{Cl}=14.96-16.36)$; malaise $(\mathrm{IR}=15.33,95 \% \mathrm{Cl}=14.65-16.04$; fatigue $(\mathrm{IR}=15.02,95 \% \mathrm{Cl}=14.35-15.72)$; abdominal pain $(\mathrm{IR}=10.18,95 \%$ $\mathrm{Cl}=9.67-10.70$ ); chest pain (IR=8.48, 95\% $\mathrm{Cl}=8.03-8.95)$; and depression ( $\mathrm{IR}=7.75,95 \% \mathrm{Cl}=7.32-8.20)$. In contrast, the 6 lowest IRs were for maculo-papular rash $(\mathrm{IR}=0.01,95 \% \mathrm{Cl}=0.00-0.04$; injection-site necrosis ( $\mathrm{IR}=0.01,95 \% \mathrm{Cl}=0.00-0.03)$; erythema multiforme $(\mathrm{IR}=0.01$, $95 \% \mathrm{Cl}=0.00-0.04)$; hypoesthesia ( $\mathrm{IR}=0.00,95 \% \mathrm{Cl}=0.00-0.02)$; StevensJohnson Syndrome $(\mathrm{IR}=0.00,95 \% \mathrm{Cl}=0.00-0.02)$; and xerophthalmia (IR=0.00, 95\% $\mathrm{Cl}=0.00-0.02$ ).
CONCLUSIONS: Study results show strong convergence between the realworld safety profile of IFN $\beta$-1a SC tiw and its U.S. label. Our findings demonstrate the value of using real-world evidence obtained from administrative claims to complement clinical trial and postmarketing surveillance data in order to characterize the safety profile of established products, such as IFN $\beta$-1a SC tiw, in the postmarketing context.

J Manag Care Spec Pharm. 2015;21(8):650-60

Copyright $\odot 2015$, Academy of Managed Care Pharmacy. All rights reserved.

\section{What is already known about this subject}

Although interferon beta-la administered subcutaneously 3 times weekly (IFN $\beta$-la SC tiw) was approved in the United States in 2002 as a first-line therapy for the treatment of relapsingremitting multiple sclerosis, its safety profile has been established primarily in pre- and postauthorization clinical trials and postmarketing surveillance-sources that may not adequately represent the context of real-world clinical care and the broader range of patients treated in actual practice.

The most commonly reported adverse events (AEs) associated with IFN $\beta$-la SC tiw in controlled clinical trials include injection site disorders, influenza-like symptoms, abdominal pain, depression, headache, elevation of liver enzymes, and hematologic abnormalities.

\section{What this study adds}

This study is unique because it describes the safety profile of IFN $\beta$-la SC tiw as characterized in its U.S. prescribing information, using real-world data from a large, longitudinal, and geographically diverse U.S. health care administrative claims dataset.

Study results show strong convergence between the real-world safety profile of IFN $\beta$-la SC tiw and its U.S. label. The relative frequency of adverse events seen in the administrative claims dataset was comparable with that reported in pivotal trials.

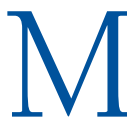
ultiple sclerosis (MS) is a chronic and progressively debilitating condition that affects the central nervous system. ${ }^{1}$ Worldwide, the disease is estimated to affect approximately 2.1 million individuals. ${ }^{1}$ During the initial disease course, approximately $85 \%$ of patients develop 
relapsing neurological symptoms, a condition referred to as relapsing-remitting MS (RRMS). While disease course and rate of progression vary widely, common symptoms of MS include numbness or paresthesia, impaired motor coordination, visual problems, bladder and bowel dysfunctions, sexual and cognitive dysfunction, and fatigue. ${ }^{2}$

Currently, various disease-modifying drugs (DMDs) are recognized as first-line therapy for RRMS. Prominent among these are the interferon betas (IFN $\beta \mathrm{s}$ ), a class of injectable products that features intramuscular (IM; Avonex [IFN $\beta$-la IM], 30 micrograms $[\mu \mathrm{g}]$ once weekly) and subcutaneous options (SC; Rebif [IFN $\beta$-la SC], $22 \mu \mathrm{g}$ or $44 \mu \mathrm{g} 3$ times weekly [tiw]; Betaseron [IFN $\beta$-1b SC], $250 \mu$ g every other day; and Plegridy [peg-interferon $\beta$-la SC], $125 \mu$ g every 2 weeks). While typically well tolerated, certain class effects of IFN $\beta$ s include anemia; aminotransferase elevations; skin reactions (e.g., injection site redness, swelling, and pain); and neutralizing antibodies. ${ }^{2,3}$

Of these IFN $\beta$ products, interferon beta-la administered subcutaneously 3 times weekly (IFN $\beta$-la SC tiw) is one of the most widely prescribed, with over 15 million doses sold since its approval by the U.S. Food and Drug Administration (FDA) in 2002. ${ }^{4}$ The effectiveness and safety of IFN $\beta$-la SC tiw have been well established in clinical trials., 5

To date, the U.S. label information on the safety profile of IFN $\beta$-la SC tiw has been derived predominantly from pivotal randomized controlled clinical trials and postmarketing surveillance. ${ }^{5,6}$ The most common adverse events (AEs) seen in controlled clinical trials were injection site disorders, influenza-like symptoms, abdominal pain, depression, elevation of liver enzymes, and hematologic abnormalities. ${ }^{5,6}$

Controlled clinical trials and postmarketing surveillance, however, have known biases with regard to drug-related safety information and the degree to which they are representative of "real-world" patients and clinical care practice. Trials conducted as part of clinical development are statistically powered to demonstrate superior efficacy, as opposed to safety, against placebo and are of limited duration and size. Moreover, clinical trial participants represent only a small, highly selective segment of the target patient population and receive treatment and follow-up under tight study protocols and closely monitored conditions. Similarly, postmarketing safety surveillance data, which rely on passive voluntary reporting methods, is limited by substantial underreporting.?

While numerous postmarketing, noninterventional observational studies involving IFN $\beta$-la SC tiw have been conducted, most have focused on drug effectiveness or adherence as opposed to safety. ${ }^{8-13}$ Others have focused on only $1 \mathrm{AE},,^{14,15}$ have had small sample sizes, or have featured relatively short follow-up periods ( $<2$ years). ${ }^{16-20}$ The limited follow-up periods are of particular concern given that MS is a disease characterized by a progressive course that typically spans many years. ${ }^{2}$
More recently, several open-label, longitudinal, observational studies of patients with RRMS treated with IFN $\beta$ s, including IFN $\beta$-la SC tiw, have reported data on a limited set of AEs associated with the use of these products. ${ }^{21-23}$ Such AEs included influenza-like symptoms, skin or injection problems, depression, headache, injection-site pain, fatigue, pyrexia, myalgia, transaminase level increased, pain in extremity, and liver function test abnormality. ${ }^{21-23}$

One untapped source of long-term, real-world safety information on IFN $\beta$-la SC tiw patients is that found in large health care insurer/payer claims datasets. Administrative claims datasets contain longitudinal information on patient prescription drug history, safety outcomes, and health care utilization in actual clinical care settings and the broader patient population and can be used to describe a range of AEs, despite the fact that some miscoding can occur. Notably, these claims datasets are increasingly being used by the FDA as part of its Sentinel Initiative to monitor the safety profile of drug products in a timely manner under real-world use conditions. ${ }^{24}$

The objective of this study was to describe the safety profile of IFN $\beta$-la SC tiw as presented in its U.S. prescribing information (PI), in a general MS population initiating IFN $\beta$-la SC tiw therapy.

\section{Methods}

\section{Data Source}

This retrospective cohort study was conducted utilizing data from the Truven MarketScan Commercial and Medicare Supplemental health care claims databases for the period between January 1, 2006, and December 31, 2012. The databases include information from commercial health insurance claims (inpatient and outpatient medical and outpatient pharmacy) and enrollment data from large employers and health plans across the United States. The MarketScan Medicare Supplemental and Coordination of Benefits (Medicare) database contains the inpatient medical, outpatient medical, and outpatient prescription drug information of retirees with Medicare supplemental insurance paid for by employers. These databases provide detailed cost, use, and outcomes data for health care services performed in inpatient and outpatient settings. The medical claims are linked to outpatient prescription drug claims and person-level enrollment data through the use of unique enrollee identifiers.

These databases are fully compliant with the Health Portability and Accountability Act of 1996 (HIPAA), meet the criteria for a limited-use dataset and contain none of the data elements prohibited by HIPAA for limited-use datasets. All data are de-identified to protect the privacy of patients and providers. Accordingly, institutional review board and ethics committee approval were not required for the study.

Overall, MarketScan covered approximately 158 million patients from 2000 to 2012. For the year 2012, it included 
approximately 51 million eligible subjects. The regional representation of this commercial claims database in the United States is as follows: Northeast, 5.44\%; North Central, 23.05\%; South, 38.61\%; West, 19.89\%; and Unknown, 2.98\%. Medicare does not track patients' regions. In the period from 2000 to 2012 , $85.3 \%$ of the subjects included in the database were commercially insured, and $6.5 \%$ belonged to Medicare $(n=10,459,149)$. Codes used in MarketScan are International Classification of Diseases, Ninth Revision, Clinical Modification (ICD-9-CM) codes for disease, Current Procedural Terminology/Healthcare Common Procedure Coding System codes, and National Drug Code numbers for drugs.

\section{Study Design}

This study was observational and retrospective with a "new start" design. "New start" refers to initial inception of patients onto IFN $\beta$-la SC tiw therapy as measured by no pharmacy claims for IFN $\beta$-la SC tiw in the 90 days prior to the index date. Outcomes included the AEs described in the U.S. PI for IFN $\beta$-la SC tiw in the Warnings and Precautions, Adverse Reactions, and Postmarketing Experience sections. ${ }^{25}$

\section{Study Sample}

The study sample consisted of patients aged $\geq 18$ years with evidence of MS ( $\geq 1$ medical claims associated with a primary diagnosis of MS (ICD-9-CM 340.XX) and $\geq 2$ prescription claims for IFN $\beta$-la SC tiw after the ICD-9-CM 340.XX diagnosis) during the study period January 1, 2006, to December 31, 2012. In addition, eligible patients were required to have no prior record of IFN $\beta$-la SC tiw treatment in the database history, a minimum of 90 days of continuous eligibility prior to IFN $\beta$-la SC tiw treatment initiation, and a minimum of 180 days of continuous eligibility after IFN $\beta$-la SC tiw treatment initiation. No power calculation was required, since the study was descriptive in nature and not designed to test specific hypotheses.

\section{Variables}

Exposure Measurement. The index date was defined as date of the first IFN $\beta$-la SC tiw prescription following an MS diagnosis. All outcomes were measured during the period following the index date. Patients were followed from index date until date of therapy switch or discontinuation, end of observation period (i.e., December 31, 2012), or end of insurance eligibility (censor date). Patients could experience more than 1 event and could be counted for 1 or more AEs. The list of codes for each DMD is displayed in Appendix A (available in online article).

Patient Characteristics. Baseline characteristics included patient demographics and health status. Postbaseline health care utilization, relapse rate, dose, and treatment adherence were also described.
Patient demographics and health status were recorded at the time of the index prescription claim. These variables included sex, age (years), history of other DMDs (yes/no), Charlson Comorbidity Index (CCI) score at baseline (mean and standard deviation $[\mathrm{SD}]),{ }^{26}$ and annualized relapse rate. Relapses were calculated using a validated algorithm that defined a MS-related relapse as consisting of either (a) a claim with an MS diagnosis in the primary position at any time during an inpatient hospitalization or (b) a claim with an MS diagnosis code in the primary or secondary position in an outpatient setting (including emergency room visits) in addition to a pharmacy or medical claim for a qualifying corticosteroid on the day of, or within 7 days after, the visit. ${ }^{27,28}$

Health care utilization was measured in terms of number of inpatient hospitalizations and emergency room admissions. Prior DMD use and comedication use (concomitant corticosteroid use: yes/no), adherence status, and average length of time on therapy were also assessed.

Treatment adherence was operationalized as the number of days of medication supplied within a refill interval in relation to the number of days in the refill interval, also referred to as the medication possession ratio (MPR) ${ }^{29}$ The MPR was calculated using the following formula: total prescription days of supply divided by [(last prescription fill date minus first prescription fill date) plus (last prescription day of supply)].

The MPR was reported as a percentage, with a cutoff of $80 \%$ or above defined as being adherent.

\section{Endpoints Collected}

Endpoints assessed included the incidence rates (IRs) for the AEs listed in the Warnings and Precautions, Adverse Reactions, and Postmarketing Experience sections of the April 2014 U.S. PI for IFN $\beta$-la SC tiw. ${ }^{25}$ An event of interest was defined as any sign or medical diagnosis recorded in the database that started any time after the index date.

Outcomes of interest for this study were described at the Medical Dictionary for Regulatory Activities (MedDRA, version 17.1) Preferred Term (PT) level for individual events and further categorized into system organ classes and then coded with corresponding ICD-9-CM codes (see Appendix B, available in online article).

\section{Data Analysis}

The study data were analyzed using SAS 9.4 (SAS Institute, Inc., Carey, NC). A descriptive profile of study participants by adherence status was conducted in terms of demographics; health status (e.g., comorbid conditions and relapse rate); prior DMDs; health care utilization; mean number of days of IFN $\beta$-la SC tiw usage; and IRs of specified AE endpoints. Frequency distributions were provided for categorical variables, and mean (SDs) were provided for continuous variables.

We conducted 3 sets of analyses in accordance with the 3 key safety information sections of the U.S. PI for IFN $\beta$-la 


\begin{tabular}{cl}
\hline TABLE 3 & Incidence Rates Per 100 Patient-Years \\
and $95 \%$ Confidence Intervals for \\
Adverse Events Listed in Adverse \\
Reactions Section of IFN $\beta$-1a SC tiw \\
Prescribing Information
\end{tabular}

\begin{tabular}{|c|c|c|}
\hline \multirow{2}{*}{\multicolumn{3}{|c|}{$\begin{array}{l}\text { Adverse Event } \\
\text { General disorders and influenza-like symptoms }\end{array}$}} \\
\hline & & \\
\hline Influenza-like symptoms & 15.65 & $(14.96-16.36)$ \\
\hline Headache & 6.94 & $(6.52-7.38)$ \\
\hline Fatigue & 15.02 & $(14.35-15.72)$ \\
\hline Pyrexia & 2.65 & $(2.42-2.89)$ \\
\hline Rigors & 1.29 & $(1.13-1.46)$ \\
\hline Chest pain & 8.48 & $(8.03-8.95)$ \\
\hline Malaise & 15.33 & $(14.65-16.04)$ \\
\hline \multicolumn{3}{|l|}{ Injection-site disorders } \\
\hline Injection-site necrosis & 0.01 & $(0.00-0.03)$ \\
\hline Injection-site reaction & 0.03 & $(0.01-0.07)$ \\
\hline \multicolumn{3}{|c|}{ Central and peripheral nervous system disorder } \\
\hline Hypertonia & 0.23 & $(0.16-0.30)$ \\
\hline Coordination abnormal & 2.08 & $(1.88-2.30)$ \\
\hline Convulsions & 1.38 & $(1.22-1.56)$ \\
\hline \multicolumn{3}{|l|}{ Endocrine disorders } \\
\hline Thyroid disorder & 5.08 & $(4.74-5.44)$ \\
\hline \multicolumn{3}{|l|}{ Gastrointestinal disorders } \\
\hline Abdominal pain & 10.18 & $(9.67-10.70)$ \\
\hline \multirow{2}{*}{\multicolumn{3}{|c|}{$\begin{array}{l}\text { Dry mouth } \\
\text { Hepatobiliary disorders }\end{array}$}} \\
\hline & & \\
\hline Hepatic function abnormal & 0.50 & $(0.41-0.61)$ \\
\hline \multirow{2}{*}{\multicolumn{3}{|c|}{$\begin{array}{l}\text { Bilirubinemia } \\
\text { Musculoskeletal and connective tissue disorders }\end{array}$}} \\
\hline & & \\
\hline Myalgia & 3.11 & $(2.86-3.38)$ \\
\hline Back pain & 4.16 & $(3.87-4.48)$ \\
\hline Skeletal pain & 0.35 & $(0.27-0.44)$ \\
\hline \multicolumn{3}{|l|}{ Hematologic disorders } \\
\hline Lymphadenopathy & 1.16 & $(1.01-1.32)$ \\
\hline Thrombocytopenia & 0.54 & $(0.44-0.65)$ \\
\hline Anemia & 6.25 & $(5.88-6.65)$ \\
\hline Psychiatric disorders & & \\
\hline Somnolence & 1.11 & $(0.96-1.27)$ \\
\hline Skin and subcutaneous tiss & & \\
\hline Rash, erythematous & 1.20 & $(1.05-1.37)$ \\
\hline Rash, maculo-papular & 0.01 & $(0.00-0.04)$ \\
\hline Renal and urinary disorder & & \\
\hline Micturition frequency & 3.50 & $(3.24-3.79)$ \\
\hline Urinary incontinence & 3.13 & $(2.87-3.39)$ \\
\hline Eye disorders & & \\
\hline Vision abnormal & 4.04 & $(3.74-4.36)$ \\
\hline Xerophthalmia & 0.00 & $(0.00-0.02)$ \\
\hline $\begin{array}{l}\text { List of adverse reactions does } \\
\text { psychiatric disorders [depressio } \\
\text { leukopenia). } \\
C I=\text { confidence interval; IFN } \beta \\
\text { taneously } 3 \text { times weekly; IR = }\end{array}$ & $\begin{array}{l}\text { presented } \\
\text { tobiliary } \\
\text { a-1a adn }\end{array}$ & $\begin{array}{l}\text { in Table } 2 \text { (i.e., } \\
\text { disorders, and } \\
\text { ninistered subcu- }\end{array}$ \\
\hline
\end{tabular}




\begin{tabular}{|c|c|c|}
\hline $\begin{array}{l}\text { Incidence Ra } \\
\text { and } 95 \% \text { CoI } \\
\text { Adverse Ever } \\
\text { Experience S } \\
\text { Prescribing Ir }\end{array}$ & \multicolumn{2}{|c|}{$\begin{array}{l}\text { Incidence Rates Per } 100 \text { Patient-Years } \\
\text { and } 95 \% \text { Confidence Intervals for } \\
\text { Adverse Events Listed in Postmarketing } \\
\text { Experience Section of IFN } \beta \text {-1a SC tiw } \\
\text { Prescribing Information }\end{array}$} \\
\hline Adverse Event & \multicolumn{2}{|c|}{$\begin{array}{c}\text { Study Participants } \\
(\mathrm{N}=8,107) \\
\text { IR }(95 \% \mathrm{CI}) \\
\end{array}$} \\
\hline \multicolumn{3}{|l|}{ Autoimmune disorders } \\
\hline Drug-induced lupus erythematosus & 0.25 & $(0.19-0.33)$ \\
\hline Autoimmune hepatitis & 0.04 & $(0.01-0.07)$ \\
\hline \multicolumn{3}{|c|}{ Blood and the lymphatic system disorders } \\
\hline TTP/HUS & 0.63 & $(0.53-0.75)$ \\
\hline Pancytopenia & 0.10 & $(0.06-0.16)$ \\
\hline \multicolumn{3}{|l|}{ Eye disorders } \\
\hline Retinopathy & 0.40 & $(0.31-0.50)$ \\
\hline Cotton wool spots & 0.27 & $(0.20-0.35)$ \\
\hline Obstruction of retinal artery or vein & 0.04 & $(0.01-0.07)$ \\
\hline \multicolumn{3}{|c|}{ Central and peripheral nervous system disorder } \\
\hline Hypoesthesia & 0.00 & $(0.00-0.02)$ \\
\hline Muscle spasms & 3.31 & $(3.05-3.58)$ \\
\hline Parasthesia & 6.69 & $(6.25-7.16)$ \\
\hline Musculoskeletal stiffness & 0.72 & $(0.60-0.85)$ \\
\hline Difficulty walking & 1.85 & $(1.66-2.06)$ \\
\hline \multicolumn{3}{|l|}{ Skin and subcutaneous tissue disorders } \\
\hline Erythema multiforme & 0.01 & $(0.00-0.04)$ \\
\hline Stevens-Johnson syndrome & 0.00 & $(0.00-0.02)$ \\
\hline Injection-site abscess & 1.71 & $(1.53-1.91)$ \\
\hline Injection-site cellulitis & 1.71 & $(1.53-1.91)$ \\
\hline Injection-site necrosis & 0.01 & $(0.00-0.03)$ \\
\hline \multicolumn{3}{|c|}{$\begin{array}{l}\text { aList of postmarketing adverse events does not include those already presented in } \\
\text { Table } 2 \text { (i.e., psychiatric disorders [depression, suicide], seizures, and hepatobiliary } \\
\text { disorders). } \\
C I=\text { confidence interval; IFN } \beta \text {-1a SC tiw=interferon beta-1a administered subcu- } \\
\text { taneously } 3 \text { times weekly; IR=incidence rate; TTP/HUS = thrombotic thrombocyto- } \\
\text { penic purpura/hemolytic uremic syndrome. }\end{array}$} \\
\hline
\end{tabular}

Among skin and subcutaneous tissue disorders, the highest IRs were for injection-site abscess and injection-site cellulitis ( $\mathrm{IR}=1.71,95 \% \mathrm{CI}=1.53-1.91$ for both). In contrast, the lowest IRs were seen for erythema multiforme, StevensJohnson Syndrome, and injection-site necrosis (IR $=0.01,95 \%$ $\mathrm{CI}=0.00-0.04 ; \mathrm{IR}=0.00,95 \% \mathrm{CI}=0.00-0.02 ;$ and $\mathrm{IR}=0.01$, $95 \% \mathrm{CI}=0.00-0.03$, respectively).

\section{Discussion}

Our retrospective analysis provides real-world, longitudinal information regarding safety outcomes listed in the Warnings and Precautions, Adverse Reactions, and Postmarketing Experience sections of the U.S. PI for IFN $\beta$-la SC tiw. A major strength of this study is that it reflects patients with MS receiving care under real-world clinical practice conditions across different geographic regions in the United States. Overall, our results show a safety profile that is consistent with the relative frequency of AEs listed in in the U.S. label for IFN $\beta$-la SC tiw.
Our study findings show that the highest AE IRs were for AEs of mild-to-moderate nature and were assumed to be clinically manageable. In particular, the highest reported IRs were for influenza-like symptoms, fatigue, malaise, and chest pain. In the clinical trial results in the U.S. PI, influenza-like symptoms are listed as the second most frequently occurring AE $(56 \%$ and $59 \%$ for IFN $\beta$-la $22 \mu \mathrm{g}$ and $44 \mu \mathrm{g}$ SC tiw doses, respectively). Other postmarketing studies have shown influenza-like symptoms to be a common AE associated with IFN $\beta$ therapy. ${ }^{21-23,30,31}$ Typically, these symptoms have been shown to abate, in terms of frequency and severity, with increased time on treatment. In particular, clinical trial results showed that $69 \%$ of patients reported influenza-like symptoms over the course of 1 to 4 years, but only 12\% did so after up to 8 years of follow-up. ${ }^{32,33}$ Similarly, in a postmarketing observational registry study, the incidence of influenza-like symptoms for patients taking IFN $\beta-1 \mathrm{a} 22 \mu \mathrm{g}$ and $44 \mu \mathrm{g}$ SC tiw ranged from $31.4 \%$ to $33.3 \%$, respectively, at 3 months versus $8.9 \%$ and $3.3 \%$, respectively, at 12 months. ${ }^{21}$ Fatigue and malaise are common symptoms associated with MS, with up to $90 \%$ of patients with MS reporting fatigue. ${ }^{2}$

In the controlled clinical trials and long-term follow-up studies for IFN $\beta$-la SC tiw, the most commonly reported AEs were injection-site disorders. ${ }^{4,5,23}$ While injection-site disorders are commonly reported AEs for injectable IFN $\beta$ formulations in general (particularly those administered SC), our results showed low IRs for this AE. ${ }^{14}$ In a 2011 study by Beer et al. (2011), the reported rates of injection-site reactions ranged from $57.7 \%$ for IFN $\beta$-lb SC to $67.9 \%$ for IFN $\beta$-la SC tiw. ${ }^{14}$ In our analysis, the low IRs for injection-site reactions may possibly be a function of clinician undercoding due to the relatively mild clinical nature of this event. Given the fact that the drug is self-administered in the home setting, many of these events may resolve spontaneously and relatively quickly, thus, negating the need to see a health care professional. In addition, patients who have been using an injectable drug may have become accustomed to injection-site reactions and may not even perceive them to be worth mentioning to a health care professional. Further support for this interpretation is evident in results from 1 of the IFN $\beta$-la SC tiw pivotal trials in which physicians rated the injection-site disorders as being mild in the vast majority of cases (in $84 \%$ of the high-dose cases and in $92 \%$ of the low-dose cases). ${ }^{5}$

In terms of more serious AEs, we found much lower IRs. In particular, the IR for depression was approximately half of that for influenza-like symptoms (IR of 7.75 vs. 15.65 , respectively) while those for suicidal ideation, suicide attempt, and suicide were comparatively even lower (IRs of $0.19,0.03$, and 0.00 , respectively). Depression is a commonly reported symptom for patients with MS in general, and cases of depression in patients receiving IFN $\beta$-la SC tiw have been linked to preexisting depressive symptoms. ${ }^{2,34,35}$ Additionally, depression, suicidal 
ideation, and suicide attempts have all been observed with increased frequency in patients using IFN compounds. ${ }^{25}$

Hepatic injury was most commonly reported in terms of increased liver enzyme levels, while the incidence of more serious events, including liver injury and hepatic failure, was notably lower. In 2 systematic reviews examining hepatic dysfunction in patients receiving IFN $\beta$-la SC tiw that used data from randomized controlled clinical trials and postmarketing surveillance studies, raised hepatic aminotransferase levels were predominately asymptomatic, tended to occur early (within the first 12 months) in treatment, and often were resolved spontaneously or with dosage adjustment. ${ }^{36,37}$

Other serious AEs, including Stevens-Johnson syndrome, TTP/HUS, drug-induced lupus erythematosus, autoimmune hepatitis, and urticaria, showed uniformly low IRs. Río et al. (2005), in an 8-year follow-up study of IFN $\beta$ patients, found 3 cases of urticaria and angioedema in those taking IFN $\beta$-la SC tiw. ${ }^{9}$

A strength of our study is that it aimed to reflect real-world use of IFN $\beta$-la SC tiw. Consistent with real-world usage, patients varied in terms of the degree of adherence to IFN $\beta$-la $\mathrm{SC}$ tiw. For the purposes of AE calculations, we included all patients who had been exposed to the product, not just those who were highly compliant.

An array of disease management strategies have been developed for many of the most common AEs reported here, including injection-site disorders, depression, and influenzalike symptoms. Care provided by multidisciplinary teams of health care professionals in conjunction with support services, such as nurse-staffed patient support call centers, offer patients access to information and advice on management of symptoms, side effects, and comorbidities. These services can be effective in mitigating AEs and assisting patients in being adherent, thereby optimizing treatment benefits while reducing treatment-related risks. ${ }^{31}$

\section{Limitations}

There are some limitations to the study associated with the use of commercial health care claims databases. ${ }^{38}$ Administrative health care claims data do not include clinical information (e.g., laboratory values and disease severity) or other measures (e.g., socioeconomic status) that could affect study outcomes. The ICD-9-CM code for systemic MS does not distinguish among different MS types (e.g., primary progressive MS, RRMS, or secondary progressive MS).

In the present study, we applied a highly specific inclusion approach, that is, we excluded patients without a diagnostic claim before an IFN $\beta$-la prescription.This is standard practice in claims databases to select patients on the basis of a diagnosis and a treatment. This practice increases the robustness of the sample with regards to the target indication and allows better estimation of disease duration. However, in some cases, using this method can lead to an underestimation of the total sample size because of missing diagnostic data in the inclusion time frame considered.

The identification of a relapse episode was based on a validated algorithm previously used. ${ }^{28}$ However, this algorithm is based on treatment received, and the threshold for intervention may vary among clinicians. Therefore, the true number of relapses may be underestimated. IFN $\beta$-la SC tiw use in health care claims data was assumed based on prescription fill date. It cannot be confirmed that a patient actually took the drug or took the drug as prescribed for the entire span of the prescription period (as measured by the days' supply calculation).

The database used consists of medical and pharmaceutical claims submitted by health care providers to insurance companies for reimbursement. Such claims are subject to possible coding errors and undercoding. Milder and more transient AEs (e.g., myalgia, artralgia, dizziness, and injection-site reactions) may be underrepresented in claims databases due to misdiagnosis or undercoding, since it is the health care provider's decision whether or not to report the event in the health care claim.

As with other database studies, a limitation of this study is that no causal relationship between exposure to the drug product and a particular AE can be definitively determined. In addition, we did not directly control for the possible confounding effects of existing comorbidities on study results. However, we did measure the CCI at baseline. Results showed that $71.7 \%$ of study participants had a CCI score of 0 (i.e., no comorbidity). Given that this was intended as a real-world descriptive study in which AE information from phase III pivotal trials and passive, postmarketing surveillance data were compared with $\mathrm{AE}$ reports from real-world clinical practice, the greater the adjustment for confounding, the less representative the study becomes in terms of the real-world experience of patients receiving IFN $\beta$-la SC tiw. As such, it is also possible that the IRs reported here were overestimated, although any such overestimation would not be expected to have a large effect on results.

The role of patient adherence to treatment is an important variable in the safety profile of IFN $\beta$-la SC tiw. However, it was not the goal of this study to address this specific question, as the purely observational design led to highly different groups of adherent and nonadherent patients with respect to nonmodifiable outcomes (gender, age, and disease severity). In such a study, emphasis should be given to modifiable outcomes, in order to provide the health care providers with operational guidance on how to improve specific adverse events. More research is currently being conducted in this area.

As a further point regarding external validity, generalizability of findings beyond the United States is limited because of differences in MS patient demographics, health care treatment practices, and diagnostic criteria. 


\section{Conclusions}

This study provides additional information concerning the safety profile of IFN $\beta$-la SC tiw as used under real-world clinical care conditions. Administrative claims database studies are important in order to assess whether safety results seen in clinical trials with selected patient groups and tightly defined clinical care procedures apply to unselected patient populations treated in daily practice. Overall, our study results support the safety profile as described in the Warnings and Precautions, Adverse Reactions, and Postmarketing Experience sections of the U.S. PI for IFN $\beta$-la SC tiw.

\section{Authors}

MEREDITH Y. SMITH, PhD, MPA, was Director, US Drug Safety Strategy and Science, and MARK SAMUELSON, MD, is Medical Director, US Drug Safety and Quality Assurance, EMD Serono, Rockland, Massachusetts. MERITXELL SABIDÓ-ESPIN, MD, is Associate Director, Global Epidemiology; ANTON TROCHANOV, MD, is Medical Director, GDS Product Leader, Neurodegenerative Disease; SANDRA GUEDES, PharmD, is Associate Director, Global Epidemiology; and Florent F. Richy, MPH, PhD, is Head of Global Epidemiology, Merck KGaA, Darmstadt, Germany. FRANK A. CORVINO, PhD, is Managing Partner, Genesis Group, Woodcliff Lake, New Jersey.

AUTHOR CORRESPONDENCE: Florent F. Richy, MPH, PhD, Merck KGaA, Frankfurter Straße 250, Darmstadt, Germany 64293. Tel.: +49 (0) 615172 64840; Fax: +49 (0) 615172 2000;

E-mail: florent-frederic.richy@merckgroup.com.
3. Cross AH, Naismith RT. Established and novel disease-modifying treatments in multiple sclerosis. J Intern Med. 2014;275(4):350-63.

4. Rebif sales report. Company data on file. Merck KGaA. 2014.

5. PRISMS Study Group. Randomized double-blind placebo-controlled study of interferon $\beta$-la in relapsing/remitting multiple sclerosis. Lancet. 1998;352(9139):1498-504.

6. Panitch H, Goodin DS, Francis G, et al. Randomized, comparative study of interferon beta-la treatment regimens in MS: the EVIDENCE Trial. Neurology. 2002;59(10):1496-506.

7. Rawlins MD. Spontaneous reporting of adverse drug reactions I: the data. Br J Clin Pharmacol. 1988;26(1):1-5.

8. Pozzilli C, Propserini L, Sbardella E, et al. Post-marketing survey on clinical response to interferon beta in relapsing multiple sclerosis: the Roman experience. Neurol Sci. 2005;26(Suppl 4):S174-78.

9. Río J, Tintoré M, Nos C, Téllez N, Galán I, Montalban X. Interferon beta in relapsing-remitting multiple sclerosis. An eight years experience in a specialist multiple sclerosis centre. J Neurol. 2005;252(7):795-800.

10. Milanese C. La Mantia, Palumbo R, et al. A post-marketing study on interferon beta $\mathrm{lb}$ and la treatment in relapsing-remitting multiple sclerosis: different response in drop-outs and treated patients. J Neruol Neruosurg Psychiatry. 2003;74(12):1689-92.

11. Trojano M, Paolicelli D, Zimatore GB, et al. The INFbeta treatment of multiple sclerosis (MS) in clinical practice: the experience at the MS center of Bari Italy. Neurol Sci. 2005;26(Suppl 4):S179-82.

12. Paolillo A, Pozzilli C, Giugni, E, et al. A 6-year clinical and MRI followup study of patients with relapsing-remitting multiple sclerosis treated with interferon-beta. Eur J Neurol. 2002;9(6):645-55.

13. Twok S, Nippert I, Scherer P, Haas J, Pohlau D, Kugler J. Immunomodulating drugs in multiple sclerosis: compliance, satisfaction and adverse effects evaluation in a German multiple sclerosis population. Curr Med Res Opin. 2007;23(6):1209-15.

14. Beer K, Müller M, Hew-Winzeler AM, et al. The prevalence of injectionsite reactions with disease-modifying therapies and their effect on adherence in patients with multiple sclerosis: an observational study. BMC Neurol. 2011;11:144. Available at: http://www.biomedcentral.com/1471-2377/11/144. Accessed June 16, 2015.

15. Tremlett HL, Yoshida EM, Oger J. Liver injury associated with the $\beta$-interferons for MS: a comparison between the three products. Neurology. 2004;62(4):628-31.

16. Limmroth V, Malessa R, Zettl UK, et al. Quality Assessment in Multiple Sclerosis Therapy (QUASIMS): a comparison of interferon beta therapies for relapsing-remitting multiple sclerosis. J Neurol. 2007;254(1):67-77.

17. Etemadifar M, Janghorbani M, Shaygannejad V. Comparison of Betaferon, Avonex, and Rebif in treatment of relapsing-remitting multiple sclerosis. Acta Neurol Scand. 2006;113(5):283-87.

18. Bonavita S, Dinacci D, Lavorgna L, et al. Treatment of multiple sclerosis with interferon beta in clinical practice: 2 -year follow-up data from the South Italy Mobile MRI Project. Neurol Sci. 2006;27(Suppl 5): S365-68.

19. Koch-Henriksen N, Sørensen PS, Christensen T, et al. A randomized study of two interferon-beta treatments in relapsing-remitting multiple sclerosis. Neurology. 2006;66(7):1056-60.

20. Haas J, Firzlaff M. Twenty-four-month comparison of immunomodulatory treatments - a retrospective open label study in 308 RRMS patients treated with beta interferons or glatiramer acetate (Copaxone). Eur J Neurol. 2005;12(6);425-31.

21. Sorenson PS, Koch-Henriksen N, Rovnborg M, et al. Immunodulatory treatment of multiple sclerosis in Denmark: a prospective nationwide survey. Mult Scler. 2006;12(3):253-64.

22. Gobbi C, Zecca C, Linnebank M, et al. Swiss analysis of multiple sclerosis: a multicenter, non-interventional, retrospective cohort study of diseasemodifying therapies. Eur Neurol. 2013;70(1-2):35-41. 
23. Hupperts R, Ghazi-Vissier L, Martins Silva A, et al. The STAR Study: a real-world, international, observational study of the safety and tolerability of, and adherence to, serum-free subcutaneous interferon $\beta$-la in patients with relapsing multiple sclerosis. Clin Ther. 2014;36(12):1946-57.

24. U.S. Food and Drug Administration. FDA's Sentinel Initiative. November 21, 2014. Available at: http://www.fda.gov/Safety/FDAsSentinelinitiative/ ucm2007250.htm. Accessed June 16, 2015.

25. REBIF (interferon beta-la), for subcutaneous injection. EMD Serono, Inc. Revised March 2015. Available at: http://emdserono.com/cmg.emdserono_us/ en/images/Rebif\%20PI_Jun2014_tcml15_19765.pdf. Accessed June 16, 2015.

26. Charlson M, Szatrowski TP, Peterson J, Gold J. Validation of a combined comorbidity index. J Clin Epidemiol. 1994;47(11):1245-51.

27. Ollendorf DA, Jilinskaia E, Oleen-Burkey M. Clinical and economic impact of glatiramer acetate versus beta interferon therapy among patients with multiple sclerosis in a managed care population. J Manag Care Pharm. 2002;8(6):469-76. Available at: http://www.amcp.org/data/jmcp/ Research-469-476.pdf.

28. Chastek BJ, Oleen-Burkey M, Lopez-Bresnahan MV. Medical chart validation of an algorithm for identifying multiple sclerosis relapse in healthcare claims. J Med Econ. 2010;13(4):618-25.

29. Steiner JF, Prochazka AV. The assessment of refill compliance using pharmacy records: methods, validity, and applications. J Clin Epidemiol. 1997;50(1):105-16.
30. Bayas A, Rieckmann P. Managing the adverse effects of interferon beta therapy in multiple sclerosis. Drug Saf. 2000;22(2):149-59.

31. Patti F. Optimizing the benefit of multiple sclerosis therapy: the importance of treatment adherence. Patient Prefer Adherence. 2010;4:1-9.

32. PRISMS Study Group, University of British Columbia MS/MRI Analysis Group. PRISMS-4: Long-term tolerability of interferon beta-la in relapsing MS. Neurology. 2001;56(12):1628-36.

33. Kappos L, Traboulsee A, Constantinescu C, et al. Long-term subcutaneous interferon-beta-la in patients with relapsing-remitting MS. Neurology. 2006;67(6):944-53

34. Mohr DC, Goodkin DE, Likosky W, et al. Treatment of depression improves adherence to interferon beta-lb therapy for multiple sclerosis Arch Neurol. 1997;54(5):531-33.

35. Patten SB, Metz LM. Interferon- $\beta$ and depression in relapsing-remitting multiple sclerosis: an analysis of depression data from the PRISMS clinical trial. Mult Scler. 2001;7(4):243-48.

36. Rieckmann P, O'Connor P, Francis GS, Wetherill G, Alteri E. Haematological effects of interferon- $\beta$-la (Rebif) therapy in multiple sclerosis. Drug Saf. 2004;27(10):745-56.

37. Francis GS, Grumser Y, Altieri E, et al. Hepatic reactions during treatment of multiple sclerosis with interferon- $\beta$-la: incidence and clinical significance. Drug Saf. 2003;26(11):815-27.

38. Strom BL. Data validity issues in using claims data. Pharmacoepidemiol Drug Saf. 2001;10(5):389-92 


\section{Postmarketing Safety Profile of Subcutaneous Interferon Beta-1a Given 3 Times Weekly: A Retrospective Administrative Claims Analysis}

\begin{tabular}{|c|c|c|c|}
\hline APPENDIX A & osure of Interest Codes & & \\
\hline Multiple Sclerosis & & & \\
\hline ICD-9-CM Code & Description & & \\
\hline 340 & Multiple sclerosis & & \\
\hline IFN $\beta$-la SC tiw & & & \\
\hline National Drug Code & Product Name & Manufacturer & Generic Name \\
\hline 44087002203 & SC-IFN $\beta$-la Rebif & EMD Serono, Inc. & Interferon beta-la \\
\hline 44087004403 & SC-IFN $\beta$-la Rebif & EMD Serono, Inc. & Interferon beta-la \\
\hline 44087018801 & SC-IFN $\beta$-la Rebidose Titration Pack & EMD Serono, Inc. & Interferon beta-la \\
\hline 44087332201 & SC-IFN $\beta$-la Rebidose & EMD Serono, Inc. & Interferon beta-la \\
\hline 44087334401 & SC-IFN $\beta$-la Rebidose & EMD Serono, Inc. & Interferon beta-la \\
\hline 44087882201 & SC-IFN $\beta$-la Rebif & EMD Serono, Inc. & Interferon beta-la \\
\hline
\end{tabular}

APPENDIX B MedDRA Preferred Terms, System Organ Classes, and Corresponding ICD-9-CM Codes for Adverse Events in Warnings and Precautions, Adverse Reactions, and Postmarketing Experience Sections of U.S. Prescribing Information for IFN $\beta$-1a SC tiwa

Outcomes of Interest

\section{ICD-9-CM Codes}

Blood and lymphatic system disorders

Leukopenia

Neutropenia

Lymphopenia

Anemia

Thrombocytopenia

Pancytopenia

Thrombotic microangiopathy

Hemolytic uremic syndrome

Thrombotic thrombocytopenic purpura

Lymphadenopathy

Central and peripheral nervous system disorders

Convulsions

Hypoesthesia

Muscle spasm

Parasthesia

Difficulty walking

Musculoskeletal stiffness

Seizures

Hypertonia

Coordination abnormal

Endocrine disorders

Thyroid disorder

Hypothyroidism

Hyperthyroidism

Eye disorders

Retinopathy

Cotton wool spots

Retinal artery occlusion

Retinal vein occlusion

Vision abnormal

Vision impaired

Xerophthalmia

\begin{tabular}{|l}
\hline 288.50 \\
\hline 288.0 \\
\hline 288.51 \\
\hline $287.3,281,282,283,284,285$ \\
\hline 284.1 \\
\hline 446.6 \\
\hline 283.11 \\
\hline 287 \\
\hline $289.3,785.6$ \\
\hline 780.3 \\
\hline 371.81 \\
\hline 728.85 \\
\hline 782.0 \\
\hline 719.7 \\
\hline 719.5 \\
\hline $340.0,345,345.0,345.2,345.3,345.4,345.5,345.9,780.30,780.31,780.39$ \\
\hline 358 \\
\hline 781.3 \\
\hline $240,241,242,243,244,245,246$ \\
\hline 243,244 \\
\hline 242.9 \\
\hline $362.0,362.1,362.2$ \\
\hline 362.83 \\
\hline 362.31 \\
\hline 362.35 \\
\hline 368 \\
\hline 264.6 \\
\hline
\end{tabular}




\section{Postmarketing Safety Profile of Subcutaneous Interferon Beta-1a Given 3 Times Weekly: A Retrospective Administrative Claims Analysis}

APPENDIX B MedDRA Preferred Terms, System Organ Classes, and Corresponding ICD-9-CM Codes for Adverse Events in Warnings and Precautions, Adverse Reactions, and Postmarketing Experience Sections of U.S. Prescribing Information for IFN $\beta$-1a SC tiw ${ }^{\text {a }}$ (continued)

General disorders and administration site

Headache

Fatigue

Pyrexia

Chills

Rigors

Chest pain

Hyperhydrosis

Malaise

Injection-site disorders

Injection-site necrosis

Injection-site reaction

Gastrointestinal disorders

Abdominal pain

Xerosthomia

Hepatobiliary disorders

SGPT increased

SGOT increased

Hepatic function abnormal

Hepatitis

Autoimmune hepatitis

Severe liver injury

Hepatic failure

Bilirubinemia

Jaundice

Immune system disorders

Anaphylactic reactions

Allergic reaction

Musculoskeletal and connective tissue disorders

Myalgia

Arthralgia

Drug-induced lupus erythematosus

Skeletal pain

Back pain

Psychiatric disorders

Depression

Suicidal ideation

Suicide

Somnolence

Suicide attempt

Renal and urinary disorders

Micturition frequency

Urinary incontinence

Skin and subcutaneous tissue disorders

Rash, erythematous

Rash, maculo-papular

Urticaria

Erythema multiforme

Stevens-Johnson syndrome

Quincke's edema

Pruritus

The incidence rate for suicidal ideation was calculated using the same ICD-9-CM code (300.9, unspecified nonpsychotic mental disorder) as that for suicide; however, suicidal ideation cases were distinguished from those classified as suicide cases based on the presence of 1 or more claims in the database subsequent to the ICD-9-CM 300.9 claim. ICD-9-CM = International Classification of Diseases, Ninth Revision, Clinical Modification; IFN $\beta$-1a SC tiw =interferon beta-1a administered subcutaneously 3 times weekly; MedDRA=Medical Dictionary for Regulatory Activities; SGOT = serum glutamic oxaloacetic transaminase; SGPT = serum glutamic pyruvic transaminase.

784.0

780.79

$780.60,780.61,780.62,780.63$

780.64

780.99

786.5

780.8

$780.7,780.79$

995.21

999.39

789.0

527.7

790.4

796.4

573.8

573.3

571.42

571

572.2

277.4

782.4

999.4, 995.0, 995.6

995.27, 995.3

729.1

719.4

710.0

733.99

724.5

296.2, 296.20, 296.21, 296.22, 296.23, 296.24, 296.25, 296.26, 296.3, 296.30, 296.31, 296.32, 296.33, $296.34,296.35,296.36,296.5,296.50,29.651,296.52,296.53,296.54,296.55,296.56,296.82,311$ 300.9

300.9

780.09

E95

788.41

788.3

695

696.2

708

695.1, 695.10, 695.11, 695.12

$695.13,695.14$

995.1 